\title{
Eurostudia
}

\section{De la mémoire médiatique des temps de guerre. Le travail de mémoire, les cadres paratextuels et les stratégies narratives dans Effroyables jardins de Michel Quint et son adaptation cinématographique par Jean Becker}

\section{Thomas Schmidtgall}

Volume 13, numéro 1-2, 2018-2019

Cultures en contact, entre régulations et représentations

URI : https://id.erudit.org/iderudit/1067283ar

DOI : https://doi.org/10.7202/1067283ar

Aller au sommaire du numéro

Éditeur(s)

Le Centre canadien d'études allemandes et européennes

ISSN

1718-8946 (numérique)

Découvrir la revue

Citer cet article

Schmidtgall, T. (2018). De la mémoire médiatique des temps de guerre. Le travail de mémoire, les cadres paratextuels et les stratégies narratives dans Effroyables jardins de Michel Quint et son adaptation cinématographique par Jean Becker. Eurostudia, 13(1-2), 245-271. https://doi.org/10.7202/1067283ar
Résumé de l'article

Dans cet article, l'auteur analyse les approches médiatiques du roman Effroyables Jardins de l'écrivain français Michel Quint (2000) et de son adaptation cinématographique (2002) par Jean Becker. L'accent est mis sur les stratégies narratives et paratextuelles utilisées pour véhiculer les ambiguïtés psychologiques de l'Histoire, présentes tant dans le roman que dans le film. L'analyse révèle une contextualisation cohérente des moyens littéraires et audiovisuels employés pour déconstruire l'idée d'une vision simpliste de la Deuxième Guerre mondiale et du temps de l'occupation allemande en France. L'auteur démontre que dans le cas du roman et du film, il s'agit de véritables lieux de mémoire franco-allemands construits à l'aide d'une imbrication unique d'événements narratifs et d'éléments intermédiaux, ainsi qu'une constellation sophistiquée de personnages et de ruptures temporelles.
Tous droits réservés $@$ Le Centre canadien d'études allemandes et européennes, 2019
Ce document est protégé par la loi sur le droit d'auteur. L'utilisation des services d’Érudit (y compris la reproduction) est assujettie à sa politique d'utilisation que vous pouvez consulter en ligne. 
De la mémoire médiatique des temps de guerre. Le travail de mémoire, les cadres paratextuels et les stratégies narratives dans Effroyables jardins de Michel Quint et son adaptation cinématographique par Jean Becker

\section{Thomas Schmidtgall}

Université de la Sarre

\section{Résumé}

Dans cet article, l'auteur analyse les approches médiatiques du roman Effroyables Jardins de l'écrivain français Michel Quint (2000) et de son adaptation cinématographique (2002) par Jean Becker. L'accent est mis sur les stratégies narratives et paratextuelles utilisées pour véhiculer les ambiguïtés psychologiques de l'Histoire, présentes tant dans le roman que dans le film. L'analyse révèle une contextualisation cohérente des moyens littéraires et audiovisuels employés pour déconstruire l'idée d'une vision simpliste de la Deuxième Guerre mondiale et du temps de l'occupation allemande en France. L'auteur démontre que dans le cas du roman et du film, il s'agit de véritables lieux de mémoire franco-allemands construits à l'aide d'une imbrication unique d'événements narratifs et d'éléments intermédiaux, ainsi qu'une constellation sophistiquée de personnages et de ruptures temporelles.

\section{Abstract}

This article analyzes the medial approach of the novel Effroyables Jardins written by Michel Quint (2000) and his adaption on screen of the same title (2002) by Jean Becker. The 
author focuses on the narrative and paratextual strategies and techniques used in the novel as well as in the movie to convey the psychological ambiguities of History. The analysis reveals a coherent contextualization of literary and audiovisual means to deconstruct the idea of a simplistic vision of World War II and the German occupation in France. The author shows how the novel's and the movie's unique conception of interlacing several narrative events and intermedial elements as well as its sophisticated constellation of characters, temporal ruptures and staging makes them veritable Franco-German memory spaces.

Le passé n'est pas libre. Aucune société ne le laisse à lui-même. Il est régi, géré, conservé, expliqué, raconté, commémoré ou haï

(Robin $2003: 27$ ).

La littérature comme forme externalisée de la mémoire collective a toujours joué un rôle pivot dans la (re)construction de la mémoire culturelle des sociétés ${ }^{1}$ (Gilzmer et Kmec 2016 : 6-7; Fischer 2000 : 15). Cette fonction de la littérature est particulièrement prégnante en France où le mythe manichéen d'une résistance globale de la société française à l'ennemi allemand pendant la Deuxième Guerre mondiale a commencé à être remis en question à partir des années 1970 (Gilzmer et Kmec 2016: 11; Vatter 2009 : 197). Comme d'autres sociétés européennes, la société française manifeste aujourd'hui de plus en plus une « hohe Selbstreflexivität

\footnotetext{
${ }^{1}$ Selon Jan Assmann, la "mémoire collective » se divise en "mémoire communicative " et "mémoire culturelle ». La mémoire collective se limite à 80 ans, voire jusqu'à 100 ans (de trois à quatre générations). Elle est transmise par la communication interpersonnelle et est caractérisée par sa référence au quotidien. En revanche, la mémoire culturelle est fixement ancrée dans le passé par des points de repères comme par exemple des événements historiques qui sont considérés comme importants pour un groupe. Souvent, de tels événements sont transmis par des institutions (école, université...) et font l'objet d'une intégration culturelle par un travail esthétique (Assmann 1988: 9-19). Suivant ce concept, la littérature et plus spécialement le roman en question ici contribuent plutôt à la mémoire culturelle.
} 
im Hinblick auf Vergangenes » (Gilzmer et Kmec 2016 : 13)² dont un exemple récent est le changement radical de la position du gouvernement par rapport aux restitutions du patrimoine africain (Savoy 2018).

L'importance d'une littérature qui est caractérisée par «le discours historique et la forte référence au présent », et en même temps par ce qu'Ansgar Nünning appelle « fiction historique autoréflexive » ou « roman métahistorique » (Nünning 1995 : 276-281, cité d'après Graeber 2008 : 241), peut être considérée comme un autre symptôme de ce développement qui témoigne d'une prise de conscience d'une histoire nationale de plus en plus remise en cause. Le roman Effroyables jardins (2000; ci-après EJ) de Michel Quint ${ }^{3}$, dont l'analyse sera au cœur de cette contribution, peut être associé à ce courant littéraire.

De la même manière, le film comme «Gedächtnismedium» («média de la mémoire») (Vatter 2009) n'a pas seulement influencé de manière notable la perception du passé, mais a également contribué à porter un «nouveau regard sur l'Histoire » (Vatter 2009: 197); c'est ce qu'a montré par exemple Le chagrin et la pitié (1971) de Marcel Ophüls, film documentaire d'importance majeure dans le contexte d'une révision de l'histoire de la collaboration en France (Vatter 2009 : 197). Cependant, le film de fiction a aussi contribué, au moins dans la même mesure, à (re)construire la mémoire collective de la société française (Vatter 2009 : 197-246). Dans ce contexte, le film est d'un intérêt particulier, puisque la fiction représente la réalité selon notre perception culturelle du monde (Schmidtgall 2014: 110), ce qui est d'autant plus intéressant si deux visions du passé se

\footnotetext{
${ }^{2}$ « [F]orte autoréflexion par rapport à son passé ».

${ }^{3}$ Michel Quint est originaire du Nord-Pas-de-Calais où il est né en 1949. Effroyables Jardins est paru en 2000 aux Éditions Joëlle Losfeld en version brochée. Dans cette première édition, le roman comporte 63 pages. D'autres éditions sont parues dans la collection «Folio» de Gallimard en 2004 et chez Pocket Jeunesse également en 2004. Dans le contexte de cette contribution, nous utilisons l'édition « Folio » de Gallimard.
} 
croisent comme dans le cas de la France et de l'Allemagne par rapport aux années 1940-1945.

Partant de ces réflexions, nous allons, dans ce qui suit, analyser les approches médiatiques spécifiques du roman Effroyables jardins de Michel Quint et de son adaptation cinématographique éponyme par Jean Becker (2002). Nous montrerons que, surtout dans le cas du roman, il s'agit d'un véritable lieu de mémoire littéraire franco-allemand ${ }^{4}$. L'accent sera mis sur les stratégies et les techniques narratives et paratextuelles utilisées pour véhiculer les ambiguités psychologiques transmises dans l'histoire et présentes tant dans le roman que dans le film. Nous prendrons également en considération les éléments intertextuels. L'objectif principal est donc une contextualisation cohérente des moyens littéraires et audiovisuels employés pour déconstruire une vision simpliste de la Deuxième Guerre mondiale et du temps de l'occupation allemande en France.

\section{L'histoire}

Aussi loin que je puisse retourner [...], les clowns m'ont déclenché le chagrin. Des désirs de larmes et de déchirants désespoirs, de cuisantes douleurs, et des hontes de paria. Plus que tout, j'ai détesté les augustes (EJ : 14-15).

Cette remarque introductive du narrateur à la première personne sert de catalyseur à un récit dans lequel Michel Quint invite le lecteur à un travail de mémoire qui utilise la fiction pour «faire la lumière sur les aspects sombres de

\footnotetext{
${ }^{4}$ En raison de sa leçon historique, le roman est devenu un « classique » de lecture au lycée (cf. CRDP de l'Académie de Créteil: "Télémaque. Littérature de jeunesse. », http://www.cndp.fr/crdpcreteil/telemaque/LP/sequence-jardin.htm [consulté le 26.01.2017]), de sorte qu'il existe déjà un bon nombre d'analyses et de fiches de lecture, par exemple: "Effroyables jardins : Résumé du livre", https://www.lepetitlitteraire.fr/analyses-litteraires/michel-quint/effroyables-jardins/resume [consulté le 26.01.2017]; "Effroyables jardins », http://www.etudier.com/fiches-de-lecture/effroyablesjardins/resume/[consulté le 26.01.2017].
} 
cette période historique»(Graeber 2008: 243). D'entrée de jeu, le narrateur introduit ainsi le clown comme motif central de ce roman à sujet historique, motif qui représente le « fil conducteur » (Graeber 2008 : 245) de l'histoire développée ultérieurement et relie « d'une part le passé du père au présent du narrateur, d'autre part l'histoire de la guerre à l'actualité politique »(Graeber 2008 : 245). Suivant cette logique, l'histoire d'Effroyables Jardins est déployée sur trois strates temporelles. Tout d'abord, la brève exposition du roman nous amène dans les années 1990 au procès de Maurice Papon, le fameux collaborateur français. Le lecteur apprend que, pendant le procès, un homme déguisé en clown a essayé d'entrer dans la salle. Cette première partie se termine déjà à la page deux avec le clown prononçant, lors de la tombée du verdict, les mots suivants : «Sans vérité, comment peut-il y avoir de l'espoir...?»(EJ: 14). Dès la page suivante, le narrateur nous emmène dans les années 1960, au temps de son enfance. Il dévoile son histoire familiale en commençant par la description de la honte qu'il éprouvait en tant qu'enfant vis-à-vis de son père, André. Nous apprenons que le rejet catégorique des clowns de la part du narrateur, tel qu'il est présenté dans le début du roman, se fonde sur la honte que celui-ci ressentait par rapport à son père qui se déguisait régulièrement en clown pour - dans le regard de son fils se ridiculiser devant un public.

Lors d'une projection du film Le pont de Bernhard Wicki à laquelle le narrateur assiste avec le cousin de son père, Gaston, celui-ci lui raconte une histoire vécue pendant la Deuxième Guerre mondiale et introduit ainsi le troisième niveau de temps. Ce récit entre parenthèses, qui commence à la page 33, se présente alors comme une histoire dans l'histoire : dans les années 1940, pendant le régime de Vichy, Gaston et le père du narrateur se livrent au sabotage d'un transformateur de la gare pour causer des ennuis aux occupants allemands. À leurs propres yeux, il s'agit moins d'un acte de résistance que d'une polissonnerie qu'ils ont commise « pour rigoler» $(E J: 34)$. Comme les deux ne se 
livrent pas aux autorités d'occupation allemandes, celles-ci prennent arbitrairement quatre hommes en otage dans une carrière d'argile et menacent de les tuer si les vrais coupables du crime ne se dénoncent pas. Mais les Allemands ne se rendent pas compte qu'en réalité ils ont déjà arrêté les auteurs du sabotage, le père du narrateur et Gaston. Les quatre détenus sont obligés de rester dans le trou d'argile et se voient confrontés à un dilemme : la seule option pour se libérer est de se dénoncer. Dans cette situation difficile, un soldat allemand - il s'agit du jeune Bernhard Wicki, le futur réalisateur de cinéma - fait preuve d'humanité en leur donnant à manger et en essayant de leur changer les idées en faisant le clown. Finalement, alors que les quatre hommes sont déjà convaincus qu'ils vont être fusillés, ils sont tout à coup libérés. Sans que les détenus l'aient su, une femme a dénoncé son mari qui a été grièvement blessé par l'explosion du transformateur. Apparemment, elle a pensé que «sa dépouille mortelle, à son homme, pouvait encore servir à quelque chose » (EJ:67). Nous apprenons plus tard dans le récit qu'il s'agissait de la future femme de Gaston, Nicole.

Le père du narrateur et Gaston sont profondément marqués par ces expériences. Ainsi, après la libération, le père décide de se déguiser régulièrement en clown pour « rendre hommage » au soldat allemand et pour « payer sa dette d'humanité » $(E J: 24)$. Après que Gaston lui eut raconté cette histoire, la perception du père par le narrateur change complètement: "Je m'étais lourdement trompé sur le compte de mon père » $(E J: 27)$. Il comprend enfin les motifs derrière le comportement de son père, le clown. De retour aux années 1990, le roman se termine la veille du dernier jour du procès de Maurice Papon et ce n'est qu'alors que le lecteur comprend que le clown qui entre dans la salle d'audience est le narrateur lui-même, qui suit la voie de son père pour le représenter lors du procès, « ainsi que Gaston, Nicole, Bernd et les autres » (EJ : 74), de même que pour rappeler le temps du régime du Vichy. 
Deux ans après sa parution, le succès du roman à thématique francoallemande ${ }^{5}$ suscite son adaptation cinématographique éponyme réalisée par Jean Becker. Le film sort à l'écran en 2003. Bien que Becker ait modifié plusieurs éléments dans son adaptation de l'œuvre, il conserve globalement l'ordre chronologique et les éléments clés de l'histoire. Quant à la réception du film, elle a été moins positive que celle du livre7; en effet, la réalisation de Jean Becker a été jugée "franchouillarde», son histoire caractérisée de «consensuelle», et les personnages décrits comme « médiocres » (Renard 2010 : 33-34, 36).

\section{Le cadre paratextuel - Fonction narrative d'un oxymore discursif}

L'histoire du roman et de son adaptation filmique est marquée par le sort tragique des personnages et avant tout par le geste humanitaire du soldat allemand. Celui-ci fait entendre une voix de réconciliation et d'humanité dans un temps d'affrontement où un regard nuancé et différencié ne semblait plus possible. Dans le roman, Michel Quint ramène ce conflit de grande échelle entre deux nations à un niveau individuel où les forces de résistance mentale de l'être humain sont présentées comme un contrepoids à la terreur.

En effet, Quint utilise une stratégie narrative sophistiquée et élaborée pour montrer les conséquences dévastatrices de la guerre et dessiner une opposition avant tout psychologique et émotionnelle. Cette opposition s'accentue à mesure que l'histoire progresse. À la fin du livre, il en résulte un effet de catharsis, mais

\footnotetext{
${ }^{5}$ L'histoire d'Effroyables jardins se poursuit dans Aimer à peine (2002), dans lequel nous rencontrons le même narrateur engagé dans une tragique histoire d'amour franco-allemande.

${ }^{6}$ Par exemple, dans le film Nicole est remplacée par Marie, une femme âgée, et Gaston s'appelle André. Le narrateur et personnage central, qui n'a pas de nom dans le livre, s'appelle Lucien dans le film.

${ }^{7}$ Pour Michel Quint, qui a gagné en 1989 le Grand prix de la littérature policière pour son œuvre Billard à l'étage et le Prix du roman policier de la plume de cristal en 2014, Effroyables jardins est jusqu'à ce jour son plus important succès. 70000 exemplaires ont été vendus dans les premiers trois mois pour atteindre le chiffre de 250000 en 2010. Le roman a été également traduit en une vingtaine de langues (cf. Nachtergaele $2010: 17)$.
} 
également une certaine consternation. Celle-ci est le résultat de l'interruption itérative du flux de la narration qui sert à prendre ainsi le contre-pied des préjugés simplistes et des modes de pensée désuets sur la Deuxième Guerre mondiale pour offrir un regard différencié sur l'Histoire. Cette interruption du flux de la narration est générée par plusieurs instruments narratifs : l'emploi d'un oxymore discursif surtout présent au niveau paratextuel, la fragmentation narrative et temporelle $\mathrm{du}$ récit et de l'histoire, et le développement psychologique des personnages. Ces dispositifs ralentissent la lecture et demandent une réflexion permanente de la part du lecteur pour établir la cohérence entre les différents niveaux (paratextuel, narratif, temporel, psychologique). Le lecteur est ainsi obligé de considérer toute la complexité politique et sociale dans laquelle se trouvaient les hommes pendant les temps de guerre. Par conséquent, l'emploi de ces dispositifs narratifs et leur cohabitation s'opposent à une vision simpliste des événements en temps de guerre.

Dans cette logique, le récit fait converger ces instruments narratifs pour appuyer le message central, qui consiste en une négation de toute confrontation belliqueuse tout en laissant transparaître un certain optimisme par rapport aux valeurs humaines telles que la solidarité et l'altruisme envers les personnes dans le besoin, mais aussi la révolte contre l'injustice et la cruauté. De plus, l'utilisation d'éléments a priori contradictoires que nous analysons ci-dessous, forme une narration cohérente dont le champ sémantique et interprétatif est limité par une technique narrative particulière qui est développée à plusieurs niveaux et qui consiste en l'union des trois instruments narratifs mentionnés ci-dessus. À cet égard, l'analyse des éléments paratextuels du roman se révèle particulièrement fructueuse (Genette 1987).

Surtout, le péritexte - pour utiliser la terminologie de Genette - correspond à l'histoire du roman. Nous y découvrons une construction récurrente de 
contradictions sémiotiques qui, entre autres, est à l'origine de l'effet d'interruption perceptive mentionné plus haut. Ces contradictions sont construites grâce à l'emploi simultané d'oxymores visuels et textuels. Ces derniers sont solidement ancrés dans le paratexte et se retrouvent dans le titre (1), sur l'image de la couverture (2) ${ }^{8}$ et dans une citation en exergue au début du livre (3). Linguistiquement, c'est par l'ancrage sémiotique récurrent des mêmes « signifiés » au moyen de «signifiants » différents que les limites interprétatives sont déterminées.

Ainsi, la première contradiction, et peut-être la plus visible, se trouve dans le titre lui-même, Effroyables jardins, que nous retrouvons aussi dans l'histoire, par le biais d'une remarque du père du narrateur quand les otages ont été envoyés dans la carrière d'argile : "Ton père, je me souviens c'est là, il a parlé de grenades et d'effroyables jardins. J'ai pas compris, il a pas expliqué. » (EJ : 42). En effet, il s'agit de la reprise d'une citation d'un poème de Guillaume Apollinaire ${ }^{9}$. Celle-ci, en reliant un adjectif et un substantif de deux champs sémantiques différents, crée un oxymore : "effroyable », une qualification contenant des sèmes négatifs, et «jardins", évoquant la nature ${ }^{10}$. Ici, la contradictio in adjecto est générée par l'opposition entre l'idée de la beauté et de la tranquillité du jardin et une notion de laideur et de terreur associée au mot «effroyable». Bien qu'il s'agisse d'une référence bien connue qui est presque un cliché, l'inconcevable ou ce qui est difficile à décrire est ici condensé par l'union de ces deux éléments sémantiques qui sont généralement considérés comme incompatibles. Leur combinaison dans le titre évoque de prime abord une certaine confusion perceptive. C'est une première indication donnée au lecteur sur la teneur de l'histoire qui sera développée dans le roman. Autrement dit, l'oxymore du titre annonce une

\footnotetext{
${ }^{8}$ Sur l'ancrage du même contenu sémantique dans le texte et l'image (Barthes 1964 : 40-51).

${ }^{9}$ Voir ci-dessous.

${ }^{10}$ La connotation contradictoire est aussi conservée dans la version allemande du livre. Le titre est traduit par Die schrecklichen Gärten (Quint 2004b).
} 
histoire de guerre qui contrecarre les idées stéréotypées associées à ces temps de nationalisme aigu et de confrontation belliqueuse.

Il est intéressant de constater que les images de la couverture, aussi bien de la maison d'édition Joëlle Losfeld que de la collection «Folio » de Gallimard, reproduisent la tension sémantique du titre, mais cette fois au moyen d'un code médiatique différent. La représentation de la contradiction se fait ici par des moyens visuels. Les deux images témoignent de deux approches indépendantes des maisons d'éditions: la première version de la couverture de l'édition originale publiée chez Joëlle Losfeld ne montre qu'un nez rouge en plastique sur un fond blanc. Déjà, dans cette première version, l'image établit un contraste en montrant le nez rouge, attribut du clown et, par conséquent, symbole ambigu de la gaieté, de la sociabilité, mais aussi de la tristesse, de la solitude et de la visée critique $^{11}$. Le contraste est souligné par le fond blanc de l'image qui évoque plutôt le vide. Les deux éléments en interaction créent une impression de solitude. En guise d'interprétation et en considérant l'histoire du roman, l'image pourrait être lue - paradoxalement - comme si le clown était la seule voix de la raison dans une époque d'irrationalité belliqueuse qu'il critique par le moyen de la dérision, tout en reprenant la tradition de la sottise comme critique des conditions politiques et sociales. C'est néanmoins la deuxième version parue chez Joëlle Losfeld qui révèle surtout une contradiction, car cette fois on a ajouté un casque de soldat qui, combiné au nez rouge, forme l'image stylisée d'un visage. Le nez

\footnotetext{
11 La figure du «clown», «paillasse» ou «auguste», s'insère dans une longue tradition culturelle occidentale qui, surtout dans sa dimension critique, remonte au moins jusqu'au sots du Moyen Âge, époque pendant laquelle "la liberté de la parole n'exista que sous les masque de la folie » (Picot 1878 : 236). Pour le XXe siècle, pensons à La Grimace de l'écrivain allemand Heinrich Böll, dans lequel le héros, Hans Schnier, échoue à jouer son rôle de clown après que son amie eut mis fin à leur relation par conformisme avec un environnement bourgeois catholique. Par conséquent, la figure du clown s'insère ici dans le contexte d'une critique de l'opportunisme par rapport à la société conservatrice de l'après-guerre en Allemagne (Böll 2001). D'une perspective transculturelle, nous pourrions établir un lien discursif entre Effroyables jardins et La Grimace, étant donné que dans les deux cas la critique de l'opportunisme politique et social se trouve au centre de la réflexion.
} 
est positionné à la place où se trouverait normalement le nez d'un visage humain. Le casque semble être placé sur la tête imaginaire du clown. La contradiction est construite ici par le casque militaire, symbole de la guerre qui est d'abord lié à la violence et à la peur, et qui évoque une impression de sérieux, et le nez rouge en plastique, symbole du clown et, par conséquent, symbole de la dérision caricaturant la confrontation hostile entre les deux nations.

L'oxymore est inhérent aux deux images et se construit par des moyens visuels tout en se servant d'autres symboles que le titre. Il ne fait pas uniquement allusion au contenu de l'histoire, mais s'insère dans une stratégie narrative. Autrement dit, l'image ne répète pas uniquement la figure de style du titre, mais précise aussi le contenu du roman. La contradiction encore abstraite suscitée par le titre se concrétise puisque le casque et le nez rouge représentent des éléments clés de l'histoire.

L'édition «Folio » a choisi une seule image qui fait référence à un discours plus complexe et intermédial : la couverture est illustrée avec un détail du célèbre tableau Soir Bleu (1914) du peintre américain Edward Hopper (cf. Renault 2012). Dans sa totalité, le tableau réaliste représente une soirée dans un café de Paris. Au centre, un clown habillé en blanc fume une cigarette. Bien qu'il soit assis à une table avec deux autres personnes, ces dernières semblent plongées dans leur conversation privée. À côté de la table une femme, debout, porte le regard vers l'horizon, peut-être sur les deux hommes, mais elle ne semble pas prêter attention au clown. Celui-ci tranche sur le fond par le blanc éclatant de ses vêtements et de son maquillage. Conformément au style de Hopper, le clown semble triste, mélancolique et isolé en dépit des gens qui l'entourent et du lieu de convivialité dans lequel il se trouve. L'oxymore se trouve ici illustré par un tableau célèbre qui représente la tristesse du clown opposée à la gaieté qui l'entoure. Bien que la couverture ne montre qu'une partie du tableau, cet élément fait référence à un 
contexte discursif plus large qui, en France, s'insère entre autres dans la tradition du personnage type du Pierrot triste (à partir du XVIe siècle) issu de la commedia dell'arte, personnage qui se languit de Colombine qui, elle, est amoureuse d'Arlequin (Storey 2014 : 3-34). Dans le tableau de Hopper, c'est le clown qui représente le sérieux et la solitude tout en incarnant une profession vouée au divertissement. Le rôle symbolique du clown de la couverture "Folio", par rapport à l'image de couverture de la maison Joëlle Losfeld, est inversé sans pourtant être en contradiction avec l'oxymore lui-même. Au contraire, les différentes images se complètent, définissant le cadre interprétatif du livre.

Le troisième élément dans ce triptyque d'oxymores paratextuels est une référence intertextuelle - une citation du poète Guillaume Apollinaire mise en exergue et reprise par le titre du livre : «Et que la grenade est touchante dans nos effroyables jardins » $(E J: 11)$. Il s'agit d'un extrait des célèbres «Calligrammes, poèmes de la paix et de la guerre 1913-1916». Apollinaire les a publiés aux Éditions Mercure de France en 1918. En tant que soldat, le poète a vécu les atrocités de la Première Guerre mondiale et a tenté de trouver une forme de représentation de ses expériences dans ses poèmes. Si nous considérons le poème en entier et son contexte historique ${ }^{12}$, nous découvrons que la phrase dans toute sa longueur renforce encore l'effet de contradiction. Il y a une opposition entre les isotopies «nature » et « guerre » : la «grenade » qui peut être en même temps le

\footnotetext{
${ }^{12}$ La version intégrale du poème est la suivante :

Les Grenadines repentantes

En est-il donc deux dans Grenade

Qui pleurent sur ton seul péché

Ici l'on jette la grenade

Qui se change en un œuf coché

Puisqu'il en naît des coqs Infante

Entends-les chanter leurs dédains

Et que la grenade est touchante

Dans nos effroyables jardins

(Apollinaire 1948 : 119).
} 
fruit et l'arme de guerre; le fruit contient un jus rouge qui, dans le contexte du poème, fait allusion au sang que l'arme fait jaillir; le mot « touchant », qui s'utilise aussi bien dans le sens de «toucher sa cible» que dans le sens d'«être émouvant». «Grenade», c'est également la ville en Andalousie, dans le sud de l'Espagne; et les enfants des rois espagnols et portugais s'appelaient «Infante », un mot qui est utilisé dans le poème.

Nous avons déjà vu ci-dessus que l'opposition inhérente au poème est également abordée dans le péritexte du roman pour traduire l'atrocité de la guerre. Mais elle se trouve aussi au niveau significatif des mots. La cohabitation des oppositions sur plusieurs niveaux (paratextuel, sémiotique, et - comme nous allons voir - aussi narratif et psychologique) forme un oxymore discursif élaboré. Sa fonction principale est de faire ressortir l'apparente contradiction par rapport aux valeurs humaines pour renforcer de manière percutante le message antiguerre du roman.

L'affiche du film reproduit essentiellement la couverture de la deuxième version de l'édition Joëlle Losfeld; le cadre paratextuel se révèle ainsi en partie identique à celui du roman. Cependant, la couverture de l'édition du film en DVD se distingue de celle du livre. Hormis le titre du film qui est complété par l'image du casque et du nez rouge en miniature, nous y découvrons au-dessus un photogramme du film qui montre trois de ses acteurs principaux : Jacques Villeret, André Dussollier et Thierry Lhermitte. La scène plutôt gaie montre les trois personnages en train de rire. Deux des personnages se serrent amicalement dans les bras. L'ambiance joyeuse est aussi ancrée textuellement par une citation du Studio magazine, un magazine de cinéma français, décrivant le film comme « un film simple, touchant, plein d'humanité et de tendresse ». Il faut souligner que cette ambiance gaie et amicale estompe le côté plus sombre de la couverture aussi reproduite sur le DVD et ne présente pas la même ambiguïté que l'affiche 
$\mathrm{du}$ film et les couvertures du roman. Bien que le casque avec le nez rouge y soit présent, la jaquette du DVD atténue les contraintes liées à la guerre.

Le film recourt donc moins à la construction d'un oxymore paratextuel que le roman : bien que celui-ci soit présent, il est moins visible. Il se peut que cela soit dû à la fonction marketing de la couverture qui consiste principalement à attirer visuellement un client potentiel et, par conséquent, vise à transmettre un seul message principal. À cela s'ajoute que le péritexte d'un livre est connecté matériellement à son contenu et qu'un livre peut être perçu dans sa totalité ${ }^{13}$ sans une machine intermédiaire (ce qui n'est pas le cas pour un DVD qu'il faut d'abord mettre dans un lecteur DVD). La fonction du paratexte dans le cas d'un livre est alors plus immédiate, ce qui est confirmé dans le cas d'Effroyables Jardins dans lequel il y a - comme nous avons vu - une forte imbrication significative de plusieurs niveaux littéraires.

\section{Fragmentations narratives et temporelles}

Dans le roman, le récit commence par une scène du procès de Maurice Papon dont le moment clé est l'apparition du clown dans la salle d'audience, pour passer ensuite à un paragraphe qui mentionne les lois de Vichy et la collaboration. Cette exposition, brève, a donc une forte connotation historique. À la fin de cette exposition, le narrateur évoque sa propre vie en nous faisant savoir que, quand il était enfant, il n'aimait pas les clowns.

La succession de ces étapes narratives qui, par leurs allusions historiques, font appel à la mémoire culturelle des Français, ainsi que le passage soudain au niveau personnel, semblent d'autant plus hâtifs qu'ils ne se produisent que dans

\footnotetext{
13 Après avoir regardé la couverture et le titre, nous pouvons immédiatement commencer à lire ou feuilleter le livre.
} 
les trois premières pages. Michel Quint révèle ici un trait caractéristique de son œuvre: une imbrication narrative d'événements et de ruptures temporelles. En effet, la suite d'événements, qui semblent incohérents et fragmentaires au début, finit par former un ensemble narratif à la fin du roman. C'est le lecteur qui est censé recomposer cet ensemble au cours de la lecture, accédant ainsi à une cohérence narrative (cf. Graeber 2008 : 249).

Les fragments narratifs se distinguent par rapport au temps de l'histoire dans lequel les actions se déroulent ou auquel la description de la situation en question se réfère. Ils se distinguent également par rapport au point de vue narratif. L'histoire est en grande partie racontée par un narrateur intradiégétique, voire autodiégétique. Cet aménagement narratif permet d'abord au lecteur de saisir le monde affectif du personnage principal et de suivre son cheminement. Pourtant, la courte description initiale du procès Papon ne se particularise pas seulement par la mise en italique, mais aussi par une focalisation externe et un narrateur extradiégétique. En outre, le récit du narrateur principal est interrompu par l'histoire métadiégétique de Gaston qui, par sa mise en abyme, ouvre une autre parenthèse $(E J: 33-71)$. Celle-ci forme le noyau central du roman et fournit l'explication au comportement du père. Le récit linéaire s'interrompt alors à plusieurs reprises et chaque fragment narratif change de narrateur, représentant, selon le cas, une analepse ou encore une prolepse par rapport à l'histoire principale.

Cette fragmentation freine la compréhension du lecteur aussi bien par rapport au temps que par rapport à la perspective. De surcroît, elle relativise sa capacité à saisir l'ensemble cohérent de l'histoire : une compréhension globale n'est possible qu'à la fin du roman. En même temps, la fragmentation narrative encadre l'histoire principale racontée par Gaston, qui représente une péripétie, et engendre un changement d'attitude du narrateur par rapport à son père. La 
découverte des expériences vécues par Gaston et le père scinde la relation pèrefils en un «avant» et un «après ». Elle est l'élément assurant la cohésion de l’histoire familiale.

Bien que le roman recoure à la fiction, le changement constant de narrateurs et de perspectives estompe la distinction entre la narration factuelle et la narration fictive. Michel Quint emploie un collage de perspectives narratives pour, d'un côté, rompre avec l'idée d'une expérience collective et uniforme de la nation par rapport à la guerre et, de l'autre, souligner les conséquences des expériences individuelles sur la société entière. Il s'insère ainsi dans une tradition de la représentation de la guerre qui oscille entre narration factuelle et fictionnelle, entre récit des événements et interprétation de ces mêmes événements. La préférence, l'accentuation ou le mélange des deux procédés narration factuelle et fictionelle - dans la narration de la guerre ont surtout évolué dans une tradition littéraire depuis la guerre de 1870-187114; Michel Quint s'en sert habilement pour transmettre un message de réconciliation, alternant les types de narrateurs et les narrations fictionnelle ou factuelle.

Le morcellement de la structure narrative reflète également la psychologie du narrateur. Le passage d'un sentiment de honte à une compréhension, voire à une fierté par rapport à son père $(E J: 24)$ n'est pas le résultat d'une lente évolution; il survient plutôt lorsque le narrateur apprend soudainement un élément important du passé de son père. Ce n'est que lorsqu'il connaît toute l'histoire de son père qu'il est capable de juger son comportement de manière adéquate. La fragmentation des étapes narratives provoque la même sensation de contradiction et les mêmes interruptions dans le flux de la narration que les oxymores analysés plus haut. De cette manière, une communication entre les

14 Voir l'article de Jörg Lehmann sur les formes et les fonctions de la narration littéraire et historiographique après 1870-1871 et 1914-1918 (Lehmann 2014). 
deux niveaux d'interprétation est créée, fût-ce de manière implicite. En effet, le lecteur est censé réunir les éléments constitutifs de l'histoire qui ne pourront être complétés qu'à la fin de celle-ci pour former une histoire cohérente. Sur le plan de la narration, la quantité d'étapes narratives relève aussi du choix de l'auteur. Le rythme du roman de Quint et la relation entre le «temps du récit » et le « temps de l'histoire » obéissent à une subdivision prononcée de sept stations narratives : 1) les années 1990 : le procès de Maurice Papon; 2) les années 1960 : la description de la situation familiale du narrateur; 3) la séance de cinéma (Le pont de Bernhard Wicki); 4) l'histoire de guerre racontée par Gaston (une analepse); 5) au cinéma après l'histoire de Gaston; 6) la mort du père, et 7) de retour au procès de Maurice Papon. Évidemment, les étapes narratives du roman ne suivent pas un ordre chronologique et la relation entre le temps du récit et le temps de l'histoire est marquée par des analepses, des prolepses et des chevauchements. Cette fragmentation temporelle correspond au rapport entre le présent et les événements du passé qui, d'un point de vue historique et personnel, ne peuvent être considérés de manière isolée : faire revivre le passé signifie aussi découvrir comment le passé influence la vie actuelle.

Pour la version cinématographique d'Effroyables jardins, le réalisateur Jean Becker a choisi une approche différente : ni le procès Papon ni les lois de Vichy n'y jouent un rôle. Pour amorcer le récit, Becker opte pour les années 1960, au moment où Lucien (l'équivalent du narrateur anonyme du roman) assiste à une séance de clown de son père, moment déclencheur de l'histoire de guerre qui changera la vie de Lucien. La comparaison des expositions du roman et du film peut aussi être considérée comme emblématique des différentes stratégies narratives. En effet, ce sont surtout les scènes initiales du roman replaçant l'histoire personnelle du narrateur dans un cadre historique plus large qui permettent au lecteur une contextualisation adéquate et donnent une visibilité concrète à l'interaction entre la guerre et le destin individuel. Dans le film, par 
contre, cette dimension est absente. Pourtant, dans le film, il y a au moins une scène très puissante qui, à la fois, représente une différence majeure entre le roman et le film et montre l'autonomie de ce dernier par rapport à la version littéraire. Contrairement au roman, le soldat allemand meurt dans le film : quand son supérieur veut l'obliger à tuer les otages, non seulement il refuse de les fusiller, mais, comme acte de résistance, il se met un nez rouge; il est alors immédiatement fusillé par son supérieur. Le soldat tombe par terre et, en gros plan, on aperçoit son sang rouge qui coule lentement sur l'argile blanche dans la carrière où sont détenus les otages.

Cette scène clé rompt avec le ton qui, jusqu'à ce moment, semblait plutôt léger, les éléments comiques étant prépondérants. L'assassinat de Bernd, le soldat-clown, par un compatriote fait voir au spectateur l'horreur de la guerre, qui ignore les notions de bien et de mal. Sur le plan narratif, c'est surtout cette scène qui reprend l'opposition sémiotique et l'effet de confusion déjà relevés dans l'analyse du paratexte et qui, dans le cas du roman, sont créés par la fragmentation narrative. Jean Becker se sert habilement d'un outil filmique à la fois simple et puissant, propre au film en tant que médium : le gros plan nous confronte au contraste du sang et de l'argile blanche, ainsi qu'au nez de clown rouge qui tombe. Le film arrive ainsi à condenser toute l'ambiguïté de l'histoire dans une seule scène qui ne se trouve pas dans le roman. C'est à ce moment-là que l'effroi se saisit du spectateur grâce au caractère d'immédiateté du film. L'interruption du flux de la narration se fait plus soudainement et plus brutalement que dans le livre; il en résulte une efficacité certaine du propos didactico-émotionnel anti-guerre. La version à l'écran confirme ainsi la réputation de l'adaptation filmique de textes littéraires comme «plurimediale 
Verarbeitungsform » et celle du film comme "größte Emotions-Maschine des 20. Jahrhunderts » (Spedicato et Hanuschek $2008: 7)^{15}$.

L'oxymore, qui dans le roman et dans son paratexte nous saute quasiment aux yeux, n'est pas aussi immédiatement visible dans le film. Une analyse plus approfondie de tout le discours filmique et de la communication inhérente entre les différents niveaux représentatifs (le péritexte, l'histoire, la mise en scène, les plans choisis...) est nécessaire pour saisir l'oxymore filmique.

Du fait de codes médiatiques différents, mais certainement aussi en raison de l'intention du réalisateur du film, la caméra joue le rôle d'un narrateur hétérodiégétique. Quand André, un ami du père qui joue le rôle du Gaston du roman, raconte ce qu'il a vécu pendant la guerre, la caméra maintient cette perspective externe. Seule la voix off d'André introduit un narrateur intradiégétique. Bien que dans le film les modes narratifs soient a priori moins complexes en ce qui a trait aux points de vue, le récit filmique tire profit d'un effet produit par les codes multiples de l'audiovisuel : l'illusion de l'expérience immédiate et, par conséquent, l'impression d'être le témoin direct de l'histoire. Cet effet résulte du fait que le temps narratif du film ne peut être représenté - au sens strict - que par des «scènes », en raison de son mode de représentation visuelle reposant sur une succession d'images. Si le film veut offrir des sommaires ou des ellipses, il est obligé de recourir à d'autres moyens tels que, par exemple, la modification des éléments de l'image, du passage des images ou l'insertion d'un texte écrit ${ }^{16}$. Cependant, ce sont en même temps ces moyens propres au film qui font naître chez le spectateur une impression de vécu direct générant une sensation d'immédiateté et de proximité. Dans la version

\footnotetext{
15 «Un média de transformation utilisant plusieurs codes médiatiques », «la machine d'émotions la plus grande du XXe siècle ».

${ }^{16}$ Dans l'adaptation de Becker, par exemple, le changement de coiffure des personnages comme signe de vieillissement nous indique l'écoulement du temps.
} 
cinématographique d'Effroyables jardins, Becker a omis plusieurs étapes narratives du roman telles que le procès Maurice Papon ou la mort du père (encore vivant à la fin du film) et il opte pour une autre approche. Bien qu'il réduise le nombre d'événements narratifs par rapport au roman, il utilise les moyens filmiques décrits ci-dessus pour reprendre la même construction d'oppositions et d'interruptions qui, dans le livre, se réalisent par le rythme narratif et qui sont aussi localisées au niveau paratextuel.

\section{La fonction du développement psychologique des caractères}

Dans son roman, Michel Quint conçoit un réseau de personnages impliqués dans une histoire de résistance qui met l'individu dans un contexte relationnel particulier : la vie du narrateur à la première personne, d'abord inconsciemment, puis, vers la fin du livre, consciemment marquée par l'histoire de son père André vécue pendant la guerre. Le destin de ce dernier n'est pas seulement lié à celui de Gaston, complice de l'acte de sabotage, mais aussi à sa femme Nicole, qui est en même temps la veuve de leur sauveteur. Indirectement, ces deux protagonistes sont responsables du fait que les deux autres otages, Henri et Émile, sont également retenus dans la carrière, parce que les Allemands ne savent pas que, parmi les détenus, se trouvent déjà les vrais coupables. Mais c'est avant tout Bernd qui change par ses gestes humanitaires - il leur donne à manger et essaie de les distraire - la situation désespérée des otages. Il contribue également à transformer leur vision de « l'ennemi », ce qui induit une gratitude profonde chez André. Ici se dévoile l'origine de l'embarras du narrateur face à l'habitude insolite de son père : en effet, André se déguise en clown pour rendre hommage à Bernd et son geste humanitaire .

Jean Becker maintient pour l'essentiel les mêmes personnages et la même constellation relationnelle, malgré de petites différences. Dans le film, Nicole, qui est la mère du narrateur dans le roman, est remplacée par deux nouveaux 
personnages : Marie, épouse de Félix; elle et lui sont déjà très âgés; et Louise, épouse de Jacques. Le cousin Gaston, qui dans le roman raconte au narrateur l'histoire vécue pendant la guerre, est remplacé par André. Dans le film, le narrateur, qui n'a pas de nom dans le roman, s'appelle Lucien. Le nom de son père - «André » dans le roman - change aussi : il s'appelle Jacques dans le film. La constellation des personnages dans le film peut être visualisée de la manière suivante :

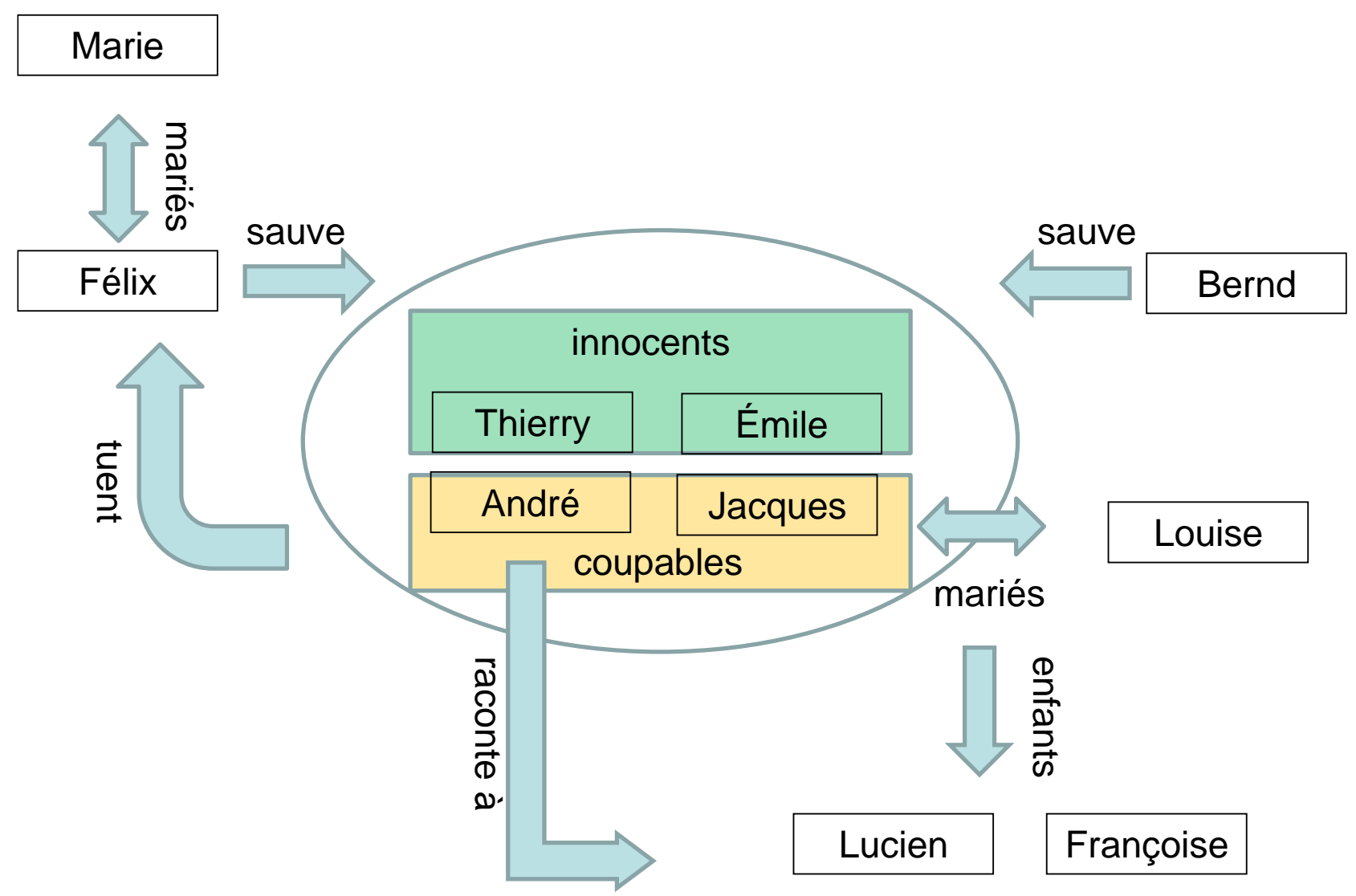

Tableau 1 : Les relations entre les personnages dans le film Effroyables jardins

Malgré ces différences, le système relationnel des personnages est le même dans le roman que dans la version filmique. Tant dans le roman que dans le film, les vraies relations des personnages ne se révèlent qu'au fur et à mesure, de 
même que la logique des étapes narratives. Les deux mécanismes littéraires - tant sur le plan des relations des personnages que sur celui du temps de l'histoire fragmenté - forment ainsi un oxymore discursif qui renvoie à la complexité des relations entre les deux pays. Analogiquement, celles-ci se passent d'une interprétation simpliste et réductrice et ne révèlent leur vraie nature qu'à $y$ regarder de près.

\section{Conclusion}

Effroyables jardins de Michel Quint est un roman à première vue simple, dont le message est clair et facile à saisir. Néanmoins, une analyse plus détaillée révèle une structure littéraire élaborée et une stratégie narrative complexe, également marquées par l'emploi particulier de moyens littéraires sophistiqués. En effet, le vif succès remporté par le livre auprès du grand public a pour une grande part été attribué au style qui «brisant avec la rigidité de la rhétorique dominante, dérangeant l'assurance de l'éloquence en place, fait résonner à l'oreille des parlures inhabituelles » (Regnier 2002 : 75). Bien qu'à première vue les pages liminaires semblent utiliser un langage simple et peu sophistiqué, nous découvrons au cours de l'histoire la complexité d'une création littéraire dont, déjà, les remarques initiales du narrateur sur les clowns laissaient entrevoir un contexte familial psychologiquement chargé, et qui ne sera dévoilé dans son intégralité que vers la fin de l'histoire. L'impact et le succès du livre découlent surtout d'une stratégie littéraire bien conçue qui se sert de moyens narratifs élaborés.

Quint rompt avec les idées et les attitudes traditionnelles de la génération de la guerre et rejette l'idée d'une opposition simpliste entre ami et ennemi. À l'aide d'un oxymore discursif qui innerve l'ouvrage entier et qui se répercute à plusieurs niveaux, il maintient une certaine tension psychologique et empêche le lecteur de faire son nid dans son premier jugement des personnages et de 
l'histoire qu'il remet continuellement en question à trois niveaux : sur le plan du cadre paratextuel et intermédial; sur le plan des étapes narratives; et sur celui du temps et des personnages. C'est surtout grâce à un paratexte élaboré dont les éléments (titre, citation, couverture...) sont complémentaires que le message central du livre est transmis, non pas uniquement de manière dénotative, mais également de manière connotative. Les différents éléments paratextuels, de même que les références intermédiales et historico-culturelles (Apollinaire, Hopper, etc.), témoignent d'une forte densité sémantique, tout en construisant déjà sur la surface paratextuelle du roman un oxymore discursif qui définit le cadre interprétatif.

Dans ce contexte, la figure clé du clown, qui se trouve bien ancrée dans le paratexte et dans l'histoire elle-même, reprend une longue tradition occidentale qui l'assimile à une figure ambivalente de l'humour, de la sottise, de la critique et de la tristesse tout en même temps. Par conséquent, le clown est mis en scène comme un instrument de la critique des temps de guerre, de l'opportunisme et des regards simplificateurs. Parallèlement, il s'insère dans un discours de contradiction établi par la narration et la structure du roman. L'impression de contradiction permanente établie par le paratexte se retrouve également dans la structure narrative qui, par sa fragmentation et ses ruptures, provoque un effet de confusion. Cette confusion joue fortement sur le lecteur et ne se résout qu'à la fin du roman, quand l'histoire de guerre vécue par le père du narrateur, ainsi que ses conséquences, sont saisies dans leur intégralité.

Cette « image complète » à la fin du roman est le résultat d'un bricolage littéraire qui demande au lecteur d'assembler les pièces du puzzle narratif. Ainsi, le lecteur est amené à découvrir peu à peu l'interdépendance des personnages et à envisager leurs liens à travers l'espace et le temps. Du point de vue littéraire, il s'agit d'un mécanisme habile qui permet au lecteur de vivre dans la fiction le 
caractère parfois contradictoire des temps de guerre, qui devient d'autant plus paradoxal lorsqu'on se place au niveau des destins individuels. L'effet d'attendrissement qui provoque la révélation finale donne accès à une autre vérité que seule la fiction est susceptible d'offrir et dans laquelle réside la qualité littéraire du travail de mémoire du roman de Michel Quint.

En somme, le roman réussit à établir un lien entre mémoire communicative et mémoire culturelle en faisant allusion à la mémoire communicative. Cette allusion consiste en une imitation de la transmission d'une histoire individuelle vécue pendant la guerre qui, en effet, se révèle fictionnelle, mais qui présente une certaine représentativité, car beaucoup de Français se souviennent d'histoires de collaboration ou de résistance appartenant à la mémoire communicative. En même temps, le roman lui-même appartient à la mémoire culturelle et peut être considéré comme un lieu de mémoire littéraire.

Tandis que le roman «tout à fait original » (Graeber 2008 : 241) de Michel Quint procède d'une conception unique par l'imbrication d'événements narratifs et d'éléments intermédiaux formant une espèce de «bricolage littéraire » singulier ${ }^{17}$, le film opte pour une stratégie narrative qui relève surtout de la mise en scène, dans la mesure où il profite de la pluralité des codes médiatiques et surtout du visuel. À première vue, Becker n'arrive pas à transformer la complexité de la narration du roman qui est basée sur l'abstraction du code de la langue. La réduction de la complexité de l'original fait apparaître le film comme un simple «Übertragung eines Inhalts aus einem Behälter (Medium) in einen anderen » (Paech 1998, cité dans Bohnenkamp 2012: 24) ${ }^{18}$. Néanmoins, une analyse plus profonde révèle que la qualité du film réside dans l'illusion de l'expérience

\footnotetext{
${ }^{17}$ Indirectement, l'auteur lui-même confirme cette observation en décrivant son travail d'écrivain comme étant « constamment dans le souci de décaler les réalités, de créer des failles dans le quotidien, d'y faire des plis, de le chiffonner, de le déchirer même. D'y installer le doute » (cité dans Regnier 2002 : 75).

18 « [T]ransfert d'un contenu d'un récipient (médium) à un autre ».
} 
immédiate, dans l'impression qu'éprouve celui qui le visionne d'être un témoin direct de l'histoire. Le réalisateur se sert habilement de cette illusion et il existe au moins une scène, la mort du clown fusillé par un soldat allemand, dans laquelle cela se manifeste avec évidence: Becker condensant le message qui, dans le roman, est développé tout au long de la narration. Il faut également souligner qu'il est nécessaire de considérer tous les niveaux discursifs pour saisir la sémiotique spécifique du film dans son intégralité.

Les deux créations, l'original littéraire et son adaptation à l'écran, représentent un travail de mémoire illustrant l'impact de la guerre, qui s'étend souvent à plusieurs générations et qui - surtout au niveau psychologique et émotionnel - est plus difficile à saisir que ne le suggère parfois le discours politique et national.

\section{Bibliographie}

Apollinaire, G. (1948). Calligrammes. Paris, Gallimard.

Assmann, J. (1988). «Kollektives Gedächtnis und kulturelle Identität ». Dans J. Assmann et T. Hölscher (dir.), Kultur und Gedächtnis. Francfort, Suhrkamp, p. 9-19.

Barthes, R. (1964). « Rhétorique de l'image ». Communications, vol. 4, p. 40-51.

Becker, J. (réalisateur), M. Quint (scénariste) et L. Becker (producteur). (2003). Effroyables jardins [Film cinématographique]. France, Ice 3 et UGC Images (producteurs délégués). 
Bohnenkamp, A. (2012). «Vorwort». Dans A. Bohnenkamp (dir.), Literaturverfilmungen. Stuttgart, Reclam, p. 9-40.

Böll, H. (1964 [1963]). La Grimace. Paris, Le Seuil.

Fischer, G. (2000). «Psychoanalyse und Psychotraumatologie». Dans W. Mauster et C. Pietzcker (dir.), Trauma. Freiburger literaturpsychologische Gespräche. Tome 19. Würzburg, Königshausen Neumann, p. 11-26.

Genette, G. (1987). Seuils. Paris, Le Seuil.

Gilzmer, M. et S. Kmec. (2016). "Einleitung : Nationale Narrative und Erinnerungslandschaften im Wandel ». Dans M. Gilzmer et S. Kmec (dir.), Histoire partagée - mémoire divisée? Erinnerungskultur in grenzüberschreitender Perspektive. Sarrebruck, Universaar, p. 1-25.

Graeber, W. (2008). «"Les parenthèses n'existent pas dans l'Histoire". Les romans métahistoriques de Michel Quint». Dans E. Arend, D. Reichardt et E. Richter (dir.), Histoires inventées. La représentation du passé et de l'histoire dans les littératures française et francophones. Francfort, Peter Lang, p. 241-251.

Lehmann, J. (2014). "Zwischen "Ich" und "Wir". Formen und Funktionen literarischen und historiographischen Erzählens nach 1870/71 und 1914/18 ». Dans W. Pyta et J. Lehmann (dir.), Krieg erzählen - Raconter la guerre. Darstellungsverfahren in Literatur und Historiographie nach den Kriegen 1870/71 und 1914/18. Berlin, LIT Verlag, p. 15-26.

Nachtergaele, V. (2010). "Michel Quint et le passé refoulé ». Nord': revue critique et de création littéraires du Nord-Pas-de-Calais, $\mathrm{n}^{\circ}$ 55, p. 17-24.

Nünning, A. (1995). Von historischer Fiktion zu historiographischer Metafiktion. t. I Theorie, Typologie und Poetik des historischen Romans. Trèves, wissenschaftlicher Verlag.

Picot, E. (1878). « La sottie en France ». Romania, t. 7, n²6, p. 236-326.

Quint, M. (2004a). Effroyables jardins. Paris, Gallimard.

Quint, M. (2004b). Die schrecklichen Gärten. Trad. E. Edl. Munich, btb-Verlag. 
Regnier, T. (2002). «Effroyables Jardins, Aimer à peine». Magazine littéraire, $\mathrm{n}^{\circ} 411$, p. 75.

Renard, P. (2010). «Effroyables Jardins, film de Jean Becker : une adaptation franchouillarde du roman de Michel Quint». Nord': revue critique et de création littéraires du Nord-Pas-de-Calais, n 55, p. 33-37.

Renault, S. (2012). "Soir bleu d'Edward Hopper». 27 décembre. http://www.grandpalais.fr/fr/article/soir-bleu-dedward-hopper-0. (consulté le 03.12.2018).

Robin, R. (2003). La mémoire saturée. Paris, Éditions Stock.

Savoy, B. (2018). «Restitutions du patrimoine africain: "Il faut y aller dans la joie" ». Le Monde, 12 janvier.

Schmidtgall, T. (2014). Traumatische Erfahrung im Mediengedächtnis. Zur Struktur und interkulturellen Rezeption fiktionaler Darstellungen des 11. September 2001 in Deutschland, Frankreich und Spanien. Würzburg, Königshausen \& Neumann.

Spedicato, E. et S. Hanuschek. (2008). "Vorwort». Dans E. Spedicato et S. Hanuschek (dir.), Literaturverfilmung. Perspektiven und Analysen. Würzburg, Königshausen \& Neumann, p. 7-8.

Storey, R. F. (2014). Pierrot: A Critical History of Mask. Princeton. Princeton University Press.

Vatter, C. (2009). Gedächtnismedium Film. Holocaust und Kollaboration in deutschen und französischen Spielfilmen seit 1945. Würzburg, Königshausen \& Neumann. 